Revue française de la traduction

\title{
Interprète pour le football ou commentateur sportif, pourquoi choisir?
}

Entretien avec Patrick Kendrick. Propos recueillis par Vanessa De Pizzol

\section{Patrick Kendrick et Vanessa De Pizzol}

\section{OpenEdition}

\section{Journals}

Édition électronique

URL : http://journals.openedition.org/traduire/800

DOI : $10.4000 /$ traduire.800

ISSN : 2272-9992

Éditeur

Société française des traducteurs

Édition imprimée

Date de publication : 15 juin 2016

Pagination : 47-54

ISSN : 0395-773X

\section{Référence électronique}

Patrick Kendrick et Vanessa De Pizzol, «Interprète pour le football ou commentateur sportif, pourquoi choisir? », Traduire [En ligne], 234 | 2016, mis en ligne le 15 juin 2018, consulté le 19 avril 2019. URL: http://journals.openedition.org/traduire/800 ; DOI : 10.4000/traduire.800 


\title{
Interprète pour le football ou commentateur sportif, pourquoi choisir? Entretien avec Patrick Kendrick
}

\author{
Propos recueillis par Vanessa De Pizzol
}

\section{Votre site vous présente comme interprète de métier, spécialisé dans} les événements sportifs. Avez-vous toujours envisagé de devenir interprète ?

Pas vraiment. J'ai fait mes études de premier cycle en langues étrangères (italien et français) à Londres, puis ai effectué un stage dans le cadre du programme Erasmus. Une fois mon diplôme en poche, j'ai eu le sentiment de ne pas maîtriser encore suffisamment ces langues. J'ai donc passé neuf mois à Brindisi, dans le sud de l'Italie, puis neuf mois à Avignon, en tant que professeur d'anglais. Après mon séjour à Avignon, je n'avais toujours pas les idées claires concernant mon avenir. J'ai alors déposé un dossier pour intégrer une école d'interprètes à Bath, en Angleterre. Ma motivation tenait en réalité à une revanche. Lors de mon séjour italien, on m'avait demandé d'assurer l'interprétation auprès d'un entrepreneur indien en visite en Italie, qui vendait des jouets artisanaux. II parlait anglais, on m'a tout naturellement demandé de traduire vers l'italien. Je n'avais aucune idée de ce métier et des compétences nécessaires : j'ai tenté le coup, et ce fut un désastre. J'ai baissé les bras au bout de cinq minutes, il s'agissait d'interpréter de l'anglais vers l'italien, c'était vraiment très difficile. Par ailleurs, je ne maîtrisais pas ce domaine, j'avais de grosses lacunes lexicales... L'ami qui m'a proposé cette mission m'a ensuite demandé si je n'avais jamais pensé à préparer un Master pour devenir interprète. Là, j'ai vraiment pensé qu'il se moquait de moi ! Nous avons fait des recherches et découvert qu'il fallait maîtriser deux langues étrangères, outre sa langue maternelle. Finalement, ma candidature à Bath a été retenue. C'est comme cela que je me suis lancé dans ce métier.

\section{Votre spécialisation dans le sport, et plus particulièrement le football, est donc venue se greffer par la suite, au cours de votre cursus ?}

Oui, pendant mes études de premier cycle, j'avais déjà participé à des journées de sensibilisation à la profession de commentateur sportif pour les aveugles, car il y avait apparemment des postes à pourvoir. En 2006 et 2008, j'ai suivi les ateliers organisés par la BBC qui recrutait dans ce domaine. Malheureusement, rien ne s'est concrétisé, c'est pour cette raison que 
j'ai décidé de partir à l'étranger. Quand je suis rentré en Angleterre, je me suis dit : je reste une année pour mes études, et tant qu'à être là, je vais essayer encore une fois, peut-être la dernière, d'entrer dans ce métier. J'ai toujours été passionné de football et c'est un domaine qui m'intéressait beaucoup. J'ai contacté un club de football local, le Bristol City. Au départ ce n'était pas évident parce qu'il y avait déjà trois personnes qui commentaient les matchs. J'ai eu de la chance, car le responsable, plutôt âgé, et en tout cas le plus expérimenté (c'était à la base un supporter de l'équipe), m'a dit : "Écoutez, ça fait vingt ans que je fais ce métier et franchement, je peux m'écarter sans problème. Tant que vous êtes là, vous pouvez faire les commentaires à ma place ". J'ai donc pu commenter quelques matchs. Au mois de janvier, je suis tombé sur une annonce d'une société à Londres qui recherchait des commentateurs en langue étrangère (français et italien). À l'époque, je faisais mes études pour devenir interprète et savais bien qu'avoir recours à quelqu'un qui n'était pas de langue maternelle italienne ou française ne représentait pas l'idéal. Mais l'envie d'entrer dans ce secteur était la plus forte. Je me suis dit : "Je vais tenter quand même, et tant qu'on me dira que ça va, que mes commentaires conviennent, je tâcherai de continuer... ". La vie est faite de coïncidences : le premier jour de Master d'interprétation, les étudiants ont eu la possibilité d'échanger avec les professeurs et interprètes de métier, et l'enseignante pour le couple de langues anglais-allemand a mentionné l'UEFA parmi ses clients. Je lui ai aussitôt demandé, peut-être un peu naïvement, comment elle avait fait pour s'introduire dans le milieu. Elle m'a répondu que l'allemand était indispensable pour être interprète de conférence au sein du comité exécutif de I'UEFA. II existait donc un cadre plus réglementaire et plus strict que celui des conférences de presse. Elle m'a appris que ce sont les clubs qui organisent les conférences de presse et choisissent directement les interprètes en s'appuyant souvent sur des agences ou des sociétés spécialisées. L'étape suivante a consisté pour moi à chercher dans cette direction, et je suis tombé sur une agence spécialisée dans l'interprétation pour le football. Mes clients sont principalement ceux qui sollicitent ses services. Pour l'instant, heureusement, dès qu'une mission concernant mes langues de travail, l'italien et le français, se présente, on fait appel à moi. J'ai toujours dit à mes profs que je voulais travailler dans le domaine du football et que les institutions européennes, ce n'était pas pour moi, car je n'avais pas le profil adéquat... Mais on m'a tout de même encouragé à postuler et un an et demi après mes études, j'ai réussi le test au Parlement, ce qui m'a offert plus d'opportunités en tant qu'interprète.

\section{Votre préférence va tout de même à l'univers du football et à ses conférences de presse?}

Si j'avais vraiment le choix, je ne ferais que cela. Mais chaque fois que j'ai l'occasion de travailler pour l'Union européenne, je trouve l'expérience très enrichissante, au contact de collègues plus expérimentés et de sujets variés. Dans le football, on a tendance à entendre des entraîneurs qui disent toujours la même chose, qui pratiquent la langue de bois, et c'est parfois 
ennuyeux... Le langage du football est un peu plus familier, je le sais, car je suis footeux moimême. À présent, j'apprends une nouvelle langue, le portugais, pour m'ouvrir plus de possibilités, pouvoir travailler plus régulièrement pour l'Union européenne, et le faire dans des conditions plus rémunératrices et confortables, notamment dans l'optique d'une vie de famille. L'apprentissage d'une nouvelle langue est un investissement sur le long terme et j'ai décidé de commencer dès maintenant pour être prêt le jour J.

\section{Pour revenir sur le choix de vos langues étrangères, quelle a été votre motivation ?}

J'ai étudié le français à l'école, c'était l'une des rares matières que je maîtrisais, j'avais de bonnes notes. J'ai également étudié l'allemand, mais ça a été plus compliqué pour moi. Avant d'entrer à l'université, on m'a conseillé de ne pas me limiter à une seule langue, les personnes maîtrisant une langue étrangère étant très nombreuses. J'ai dû choisir entre l'allemand et une nouvelle langue, et j'ai décidé de me mettre à l'italien. J'ai commencé l'italien à l'université, sans savoir à quoi m'attendre, et au fur et à mesure l'Italie et sa langue sont devenues une passion : à l'heure actuelle je passe beaucoup de temps dans ce pays, ma fiancée est napolitaine, mon domicile professionnel se situe à Milan. Après plusieurs années de travail à Londres, j'ai décidé de déménager à Milan. J'ai passé un an et demi là-bas et j'ai trouvé du travail en tant que commentateur sportif en anglais pour le championnat italien. Ce n'est pas un véritable choix, ça tient un peu du hasard, mais en fin de compte, ça me convient parfaitement. Le portugais, c'est un peu comme pour l'italien, c'est avant tout une passion... Je pense qu'il est pratiquement impossible de maîtriser une nouvelle langue sans une véritable vocation. L'espagnol aurait peut-être été un peu plus utile, par exemple pour m'ouvrir les portes de I'ONU, mais voilà, j'avais envie de m'installer au Portugal, où j'ai élu domicile à titre personnel. Je suis en quelque sorte le bourgeois anglais typique : j'y ai passé des vacances quand j'étais gamin et je n'aimais pas le fait de ne pas pouvoir m'exprimer en portugais. Je sais maintenant que le processus d'apprentissage prendra plusieurs années : il faut du temps pour maîtriser suffisamment une langue et travailler correctement en cabine, ce qui est mon objectif.

\section{Vous avez maintenant acquis une certaine expérience. Comment se passent les interviews avec les fortes personnalités médiatiques du milieu footballistique?}

Je pense qu'être interprète ressemble parfois au métier d'acteur, je ne suis pas le premier à le dire. II faut rester neutre, tout en restituant le plus fidèlement possible les propos de la personne. C'est vrai qu'on rencontre parfois de fortes personnalités avec lesquelles on doit vraiment faire profil bas. II est indispensable de garder à l'esprit que vous n'êtes là que pour traduire, même s'il y a des caméras. D'un côté, vous jouez un rôle très important, car sans vous personne n'arrive à communiquer, mais de l'autre, il faut prendre en compte la présence des journalistes, des entraîneurs et des joueurs qui, eux, sont vraiment des stars. Par moments, 
ce n'est pas évident : ce sont des gens toujours très pressés, et la plupart des conférences de presse se déroulent en mode consécutif. Le principal problème est que ces entraîneurs ont toujours envie de quitter la salle au plus vite pour reprendre l'entraînement. II faut tout traduire, mais de manière concise et assez rapide, ce qui n'est pas forcément très facile, car on est obligé de travailler dans les deux sens. Personnellement, je me trouve plus à l'aise quand je travaille de l'italien vers l'anglais ou du français vers l'anglais. Comme ce n'est pas pratique d'embaucher deux interprètes, et que les missions sont très intenses (de vingt à quarante minutes), j'ai dû très vite m'habituer à traduire vers le français. Heureusement, je connais très bien le domaine du football. Bien sûr, j'aimerais un jour interpréter aussi bien vers le français que vers l'anglais. Je voudrais aussi travailler avec un collègue français lors des conférences de presse, mais comme je vous l'ai dit, on n'a recours qu'à un seul interprète.

\section{Laurent Laget, traducteur spécialiste du sport, définit Mourinho comme le " cauchemar des interprètes ". II existe en effet des vidéos d'interviews de Mourinho très cruelles pour les interprètes qui ont eu affaire à lui. Avez-vous personnellement eu l'occasion de le rencontrer, de l'interviewer? Que pensez- vous de son profil un peu particulier dans l'univers du football ?}

Je l'ai croisé deux fois. J'ai eu cette chance, car on dépend toujours du tirage au sort pour les matchs européens. Chelsea a joué contre le PSG trois années d'affilée, et je suis allé à Londres à chaque occasion pour traduire les conférences de presse. Les deux premières années, c'était Mourinho, avant que Guus Hiddink ne prenne sa succession, en décembre 2015. L'entraîneur portugais a toujours été mon idole.

On dit que Mourinho a commencé sa carrière en tant que traducteur ou interprète, d'ailleurs on utilise souvent ces deux termes indifféremment, ce qui gêne certaines personnes. En réalité, son rôle était plus large. II était présent aux côtés de Bobby Robson à l'époque, l'entraîneur anglais des débuts du Sporting de Lisbonne, avant de passer au FC Barcelone. II était interprète, mais ne traduisait pas forcément ce que l'entraîneur disait : il connaissait parfaitement les questions piège et essayait de détourner un peu le sens pour éviter à l'entraîneur de tomber dans le panneau.

Mourinho maîtrise très bien l'espagnol, l'anglais, le français et l'italien, qui s'ajoutent au portugais, sa langue maternelle. Il donne souvent des interviews à la télévision française après les matchs, et j'ai remarqué qu'en conférence de presse - je ne sais pas si c'est quelque chose qu'il a mis au point avec son attaché de presse - il ne répond jamais directement aux questions qui lui sont posées en français. II attend que vous traduisiez la question en anglais et, à partir de là, il peut répondre. II écoute très attentivement ce que vous dites, car il comprend vraiment tous les termes utilisés : on dirait qu'il est en train de vérifier vos propos. Ça peut parfois donner les vidéos que vous évoquiez... Dans ces cas-là, malheureusement, je pense 
que les interprètes ne sont pas vraiment qualifiés. C'est une situation courante dans le football : on fait souvent appel à des supporters, ou à des gens qui parlent plusieurs langues et qui connaissent le football, mais qui n'ont pas forcément suivi une formation appropriée. Certains s'en sortent très bien, d'autres un peu moins. Mourinho choisit précisément les mots qu'il emploie, chaque propos est pesé, rien n'est laissé au hasard. Il est un peu à part, c'est vrai, mais il est très respectueux du travail bien fait et vous serre la main s'il est satisfait. Pour ma part, j'ai eu deux expériences plutôt positives avec lui et honnêtement, j'étais un peu triste de ne pas le croiser cette année.

\section{Quelle est votre façon de faire lorsque vous intervenez en tant qu'interprète dans} les conférences de presse ? Sur votre site, on vous voit prendre des notes très calmement pendant le discours de l'entraîneur, puis restituer parfaitement ses propos en anglais. Vous arrive-t-il de procéder différemment, sans notes par exemple?

Ça dépend. Certains entraîneurs ont tendance à faire des réponses très courtes; dans ce cas, inutile de tout noter. Mais il faut toujours rester sur ses gardes. Laurent Blanc, par exemple, a l'habitude de parler pendant trois ou quatre minutes d'affilée, et dans ce cas-là, la prise de notes devient indispensable. Hiddink, un Néerlandais qui parle très bien anglais, a tendance à faire des réponses de vingt à trente secondes, ce qui signifie que vous commencez à prendre des notes sans aller jusqu'au bout... Pour moi, la prise de notes est une manière de me donner du temps, pour pouvoir trouver le mot qui convient, avant même de commencer à traduire. II s'agit juste d'un support pour guider ma réflexion et me souvenir de ce qui a été dit. La prise de notes me vient de ma formation, et je la juge d'autant plus indispensable que dans le domaine footballistique, on travaille souvent en mode consécutif.

\section{Même si vous connaissez les personnes que vous interprétez, même si vous vous attendez plus ou moins à ce qu'elles vont dire, vous prenez toujours des notes?}

Oui, dès qu'une réponse dépasse les trente secondes, il faut passer à la prise de notes. Pour ma part, je commence à écrire juste après la première phrase, car si la réponse va au-delà des trente secondes, je ne veux pas rater la première partie de la phrase. II faut être très réactif, commencer à traduire dès que la personne a fini de s'exprimer, car les journalistes ont tendance à utiliser le moindre temps mort pour poser d'autres questions, et l'attaché de presse doit intervenir. II m'est déjà arrivé de couper quelqu'un pour dire : " Excusez-moi, je dois quand même traduire ce qu'on vient de dire "... Sinon, les questions et les réponses non traduites s'accumulent et on ne peut pas travailler correctement. 


\section{Y a-t-il des situations, drôles ou difficiles, qui ont particulièrement marqué votre expérience d'interprète?}

J'ai connu une situation vraiment inhabituelle avec l'ancien président de l'Inter de Milan qui avait décidé de quitter la présidence le jour de la conférence de presse. L'entraîneur était vraiment sous pression. La situation était tendue, ce n'était pas évident à gérer. L'entraîneur voulait absolument en découdre avec les journalistes. Le rythme était très rapide : questions et réponses se succédaient sans pause. Nous étions complètement sortis du cadre et moi j'essayais de tout y faire rentrer en faisant une petite synthèse de ce qui avait été dit. Mais l'attaché de presse m'a dit que ce n'était pas la peine, que les journalistes étrangers n'étaient pas au courant et que ce problème ne les intéressait pas. Les altercations sont toujours des moments pénibles à gérer, mais qui restent propres au domaine footballistique. En cabine, je n'imagine pas devoir crier ou insulter quelqu'un. II faut donc essayer de rester très calme et de dire exactement ce qui a été dit, même aux dépens de la qualité linguistique.

\section{Dans cet univers très particulier des conférences de presse, l'attaché de presse semble être le véritable chef d'orchestre qui vous dit si vous avez, ou non, le droit de traduire ?}

On est censé tout traduire, mais le véritable client, c'est le club qui joue à domicile. L'attaché de presse est le responsable de la communication, si l'entraîneur a dit une bêtise ou donné une réponse susceptible de créer la polémique, il peut parfois vous demander de ne pas traduire, pour calmer le jeu. II faut donc faire attention à lui, à la personne qui s'exprime, aux journalistes... On a très peu d'alliés dans la salle dès qu'on commence à interpréter.

Nous avons constaté que votre métier d'interprète spécialisé dans les conférences de presse pour le football s'articule autour de règles très strictes de tours de parole, de protocole et, parfois, de " censure " orchestrée par l'attaché de presse. On imagine combien ces missions peuvent être contraignantes et combien votre marge de manœuvre est réduite. Qu'en est-il quand vous endossez le rôle du commentateur sportif ? Vous sentez-vous plus libre de développer votre imaginaire linguistique?

Ce sont deux fonctions complètement différentes. D'une part, quand on commente un match, on peut dire tout ce qu'on veut - sans aller jusqu'à insulter les joueurs, bien sûr. Mais on peut faire des commentaires sur le système tactique, sur l'état de forme d'une équipe ou d'un joueur, sur les résultats récents des uns et des autres, etc. En revanche, en tant qu'interprète, il faut vraiment traduire tout ce qui est dit, et c'est peut-être plus facile parce qu'on sait exactement ce qu'on a à faire, le cadre étant assez strict, il suffit de trouver le ton et les mots justes, et de traduire de manière rapide et efficace. 


\section{Les deux aspects se complètent-ils et vous aident-ils à être plus efficace en tant que commentateur sportif et plus performant en tant qu'interprète ?}

Oui, en fait mon rêve serait de pouvoir commenter de belles affiches de Premier League tout en continuant à assurer des missions à Bruxelles, Milan ou ailleurs... L'idéal serait de faire à peu près deux missions par semaine pour les conférences de presse sur deux jours et deux matchs par week-end. Mais j'ai parfois l'impression de ne pas réussir à faire de véritables progrès dans les deux domaines, dans la mesure où il faudrait consacrer tout son temps à l'un des deux métiers pour devenir vraiment très bon... J'espère simplement ne pas avoir à choisir définitivement, car j'aimerais poursuivre ces deux métiers, qui sont complémentaires selon moi.

\section{Pour revenir à vos premiers pas en tant que commentateur sportif, avez-vous vraiment fait vos premières armes pendant les matchs disputés avec vos cama- rades?}

Récemment, mes camarades m'ont rappelé l'anecdote du match de tennis que j'avais disputé contre un copain. J'étais en train de perdre, il avait remporté les deux premières manches... J'ai donc décidé de commenter pendant l'échange, ce qu'on ne fait jamais à la télévision, les règles sont plutôt strictes en la matière. Au football, on peut commenter à n'importe quel moment, mais pas au tennis. Ce n'était donc pas très fair-play de ma part : je commentais les échanges au fur et à mesure, mon adversaire s'est déconcentré et j'ai finalement remporté le match en cinq manches. Du reste, la manie du commentaire sportif m'est venue très tôt. Je me souviens qu'à sept ou huit ans je jouais tout seul au football dans le jardin et que j'inventais les commentaires... À quinze ans, j'ai dit à mes parents que je voulais devenir commentateur sportif. Ils m'ont soutenu malgré les difficultés évidentes, même s'ils tenaient à ce que je fasse des études universitaires, pour avoir réellement le choix... Au final, je n'ai pas choisi entre commentateur et interprète, puisque je pratique ces deux métiers !

\section{Êtes-vous joueur de foot et, si oui, quel est votre niveau ?}

J'ai joué dans l'équipe universitaire ; il y avait un bon niveau, mais on était très loin du jeu professionnel. Certains étaient en centre de formation, mais ils n'ont pas fait carrière. Je n'ai jamais envisagé de devenir joueur professionnel, j'étais gardien de but et j'ai toujours pris beaucoup de plaisir à pratiquer ce sport. Lors de mes séjours à l'étranger, j'ai continué à jouer : j'ai réussi à trouver une équipe à Avignon, je joue ici au Portugal, j'ai joué en Italie, ce qui m'aide dans mon métier parce qu'on s'imprègne de tout le lexique, d'une façon très concrète. Quand j'arrive à l'étranger, et qu'il s'agit d'un pays qui compte beaucoup de supporters, je sais déjà que je pourrai échanger sur le football... 


\section{Selon vous, est-ce indispensable de pratiquer le sport dans lequel on travaille pour être un bon interprète ou un bon commentateur sportif ?}

Non, je ne crois pas. II faut tout d'abord comprendre le jeu, ce qui est tout à fait possible sans le pratiquer soi-même. On distingue deux rôles : celui de commentateur sportif, qui décrit ce qui se passe sur le terrain, et l'ancien professionnel, qui est présent en tant que conseiller technique. Pour le métier d'interprète, je pense qu'il n'est pas nécessaire d'avoir une connaissance approfondie du sport ou du jeu, en dépit de la tendance de certains entraîneurs à entrer dans les détails techniques. En Angleterre, on n'a pas vraiment cette culture tactique chez les journalistes, ils préfèrent poser des questions sur d'autres sujets. En Italie, au contraire, on plonge vraiment dans les spécificités du travail de l'entraîneur, et dès qu'il commence à parler de son métier, l'interprète doit être capable de déchiffrer tout ce qu'il dit, sinon cela devient très compliqué.

\section{Vous avez également commenté du tennis, quelle part ce sport occupe-t-il dans votre métier à l'heure actuelle ?}

À l'époque où je travaillais à Londres, on couvrait aussi bien les tournois de tennis que les championnats de football. Pour être honnête, je n'aime pas trop commenter le tennis, car on n'est jamais sûr de la durée du match ! II peut s'achever en deux manches, comme en cinq. Une chose est sûre : avec le tennis, on ne peut jamais dire à ses amis : " Je devrais finir vers vingtet-une heures, retrouvons-nous ensuite pour boire un coup ". Pour un match de football, en revanche, on peut!

J'ai commenté des matchs de tennis parce qu'on me l'avait proposé, tout au début de ma carrière de commentateur sportif, mais j'ai abandonné depuis. De cette brève expérience, je retiens qu'il est toujours enrichissant de pouvoir commenter plusieurs sports. Mais le football reste ma passion, et ma discipline fétiche !

kendrickpatrick86@gmail.com

http://www.patrickkendrick.com/ 